\title{
Successful treatment of dysfunctional uterine bleeding using laparoscopic bilateral uterine artery ligation
}

Received: 29 August 2005 / Accepted: 23 December 2005 / Published online: 21 February 2006

(C) Springer-Verlag Berlin / Heidelberg 2006

\begin{abstract}
We present a case of dysfunctional uterine bleeding in a woman who wished to retain her fertility but found conventional treatment neither suitable nor successful. Her condition was successfully treated with laparoscopic bilateral ligation of the uterine artery.
\end{abstract}

Keywords Dysfunctional uterine bleeding .

Menorrhagia $\cdot$ Bilateral uterine artery ligation $\cdot$ Anaemia

\section{Introduction}

Menorrhagia accounts for about $15-30 \%$ of referrals to gynaecologists. Most women have no detectable pelvic pathology and are diagnosed as suffering with dysfunctional uterine bleeding (DUB). The condition often impacts the sufferer's quality of life, and it can occasionally be associated with significant morbidity. Its aetiology is poorly understood, and there are medical, surgical, and combined methods of treating it. The treatment choice depends on the severity of bleeding, the woman's reproductive desire, and the treatment options available at the care site. The mainstays of conservative treatment are drugs, often contraceptives, and the Mirena coil. The drugs can be poorly tolerated because of their side effects and are of little use in patients trying to become pregnant. Hysterectomy remains the most curative treatment for DUB, although more endometrial ablations are now being done. The most commonly performed surgical options are also not fertility sparing, although they are not necessarily sterilising. Therefore, current treatment modalities have little to offer those women with DUB who wish to concurrently conceive.

\footnotetext{
J. S. Y. Lo $(\bowtie) \cdot$ A. Pickersgill

Women's Unit, Stepping Hill Hospital,

Poplar Grove,

Stockport, SK2 7JE, UK

e-mail: jennylo@doctors.org.uk

Tel.: +44-161-4195534

Fax: +44-161-4195582
}

We report a patient in whom none of the conventional treatments was suitable or successful. Her condition was successfully treated by ligating both uterine arteries. This is the first case in which the procedure was performed laparoscopically for dysfunctional uterine bleeding and had a successful outcome.

\section{Case report}

$\mathrm{CH}$ was a 34-year-old woman referred for a second opinion. She had had debilitating menorrhagia and dysmenorrhoea for several years and had been advised that she required a hysterectomy. She had started her periods at the age of 11 and had subsequently suffered with anaemia. This was chronic and responded poorly to iron supplements, which she had taken since she was 15 years old. Her periods were also extremely painful; she suffered from severe central menstrual cramps. The combination of the dysmenorrhoea and menorrhagia was debilitating.

After 12 years of managing her condition in primary care, she was finally referred to her local gynaecologist. She was investigated, including having a diagnostic laparoscopy, and was diagnosed as suffering with DUB. She was then treated with continuous combined oral contraceptive pills for the next 3 years. At the age of 30 she decided to start a family and stopped taking the oral contraceptives. Not surprisingly, her dysmenorrhoea, menorrhagia, and coexisting anaemia promptly returned. Her general practitioner suggested a course of tranexamic acid, with no apparent benefit. She was reviewed by her gynaecologist, and an abdominal hysterectomy was advised. At that point she asked for a second opinion.

At the time of referral for her second opinion, she was having a regular menstrual cycle, bleeding heavily for 8 days every month. Her haemoglobin was $10.6 \mathrm{~g} / \mathrm{dl}$, with a mean cell volume of $78.8 \mathrm{fl}$ and serum ferritin of $1 \mathrm{ug} / 1$. Her luteal-phase progesterone levels suggested ovulation. Clinical examination was normal. Because she was also trying to conceive, the options of further contraception pills, progestogens, the Mirena coil, endometrial ablation, 
and hysterectomy were excluded. It was suggested that bilateral uterine artery ligation might be a solution to treat her menorrhagia and preserve her fertility, and it was proposed that this be done laparoscopically. After due consideration, she opted for the surgery.

Therefore, bilateral uterine artery ligation was performed laparoscopically. The procedure was performed under general anaesthesia with the patient in the dorsolithotomy Trendelenburg position. A uterine manipulator was introduced, and videolaparoscopy was performed using a 10-mm 0-degree laparoscope inserted using the open technique through an umbilical incision. Two 5-mm side ports were inserted under direct vision lateral to the inferior epigastric vessels. Using monopolar diathermy $(90 \mathrm{~W}$ cutting current and $60 \mathrm{~W}$ coagulation) with microscissors and graspers, the peritoneum of the anterior leaf of the right broad ligament, between the round ligament and the infundibulopelvic ligament, was incised. The lateral umbilical ligament was recognised. Following this upwards, the uterine artery was identified crossing the ureter. After sharp and blunt dissection, the ureter was dissected laterally, away from the uterine artery, which was isolated and occluded with Ligaclips and then coagulated. The procedure was then repeated on the left side. The peritoneum was left open, and no antiadhesive preparations were available for use. The patient's postoperative progress was uneventful.

When she was reviewed a year later, her menorrhagia was subjectively cured, and she was satisfied with the procedure. She was no longer anaemic or taking iron supplementation. Her haemoglobin was $11.7 \mathrm{~g} / \mathrm{dl}$ with a mean cell volume of $83.9 \mathrm{fl}$. However, the procedure had done nothing for her dysmenorrhoea, which persisted and required multiple analgaesics. Further treatment options were discussed, and she subsequently had a laparoscopic presacral neurectomy that finally relieved her dysmenorrhoea. No adhesions were identified at this laparoscopy, and her peritoneum had healed as normal.

\section{Discussion}

The main blood supply to the uterus is derived from both of the uterine arteries, with the vaginal and ovarian vessels supplying approximately $58 \%$. Uterine artery embolisation is now an established and successful treatment for uterine fibroids, though it can be associated with complications and is currently not recommended as a treatment for women wishing to preserve their fertility. However, successful pregnancies have been reported following it.

The effect of surgical ligation of the uterine vessels may not be equivalent to embolisation, where more diffuse damage to the uterine vasculature is thought to occur [1]. As a result of embolisation, there appears to be an abrupt and severe cell ischaemia, with a slow and gradual cell ischaemia following ligation. Some authors recommend destroying the anastomotic vessels between the uterine and ovarian arteries to diminish the development of collateral circulation that could render the surgery less effective [2].
However, most authors who have described laparoscopic coagulation or ligation of the uterine vessels had selected patients not desiring further pregnancies.

Bilateral uterine artery ligation was first described as a treatment for postpartum haemorrhage in 1952 by Waters in a series of eight patients [3]. The largest series was initially reported in 1966 [4], with further additions increasing the numbers to 90 by 1974 [5]. In these cases, bilateral uterine artery ligation was used successfully to treat severe haemorrhage at the time of caesarean section. More recently, it has been effectively used to manage delayed postpartum haemorrhage [6] and in caesarean myomectomy [7].

The procedure was first described in gynaecological practice in 1964 to treat menorrhagia arising in association with fibroids and also with DUB [8]. Furthermore, it has been advocated to be of value in conjunction with myomectomy, both as an aid to reduce intraoperative bleeding and then to prevent the recurrence of heavy periods. Early case series reported substantial relief of symptoms following the procedure. Despite these reports, the practice of uterine artery ligation at laparotomy has been adopted by only a few gynaecological surgeons, and it has never been recognised as a mainstay treatment.

In recent times with the advent of better optics and instrumentation, laparoscopic techniques have flourished, and techniques of occluding the uterine vessels have been successfully adapted to this approach. To perform laparoscopic uterine artery ligation, the surgeon must be equipped with the necessary laparoscopic skills and should be able to precisely locate the uterine artery. The exact site at which the uterine artery is exposed and occluded is generally near its origin from the internal iliac artery [8-10]. It can also be taken nearer the uterus, although technically this is thought to be a more difficult site for isolating it [11]. How best to occlude or ligate the vessel is a matter of conjecture. Sutures were originally used, and recanalisation was later noted [4]. Similar findings occurred after bipolar desiccation [10], so some authors recommend Ligaclips, often in combination with bipolar coagulation $[9,10,12,13]$.

Laparoscopic bilateral uterine artery ligation has been used prior to laparoscopic myomectomy in various centres. A recent study by Liu et al. [10] has shown laparoscopic bipolar coagulation of uterine vessels alone, without myomectomy, to be an effective method for treating patients with fibroid-related menorrhagia and pelvic pain. It is safe and effective even in the presence of altered pelvic anatomy. The combination of laparoscopic bilateral artery ligation and intraamniotic methotrexate injection appears to be effective in preventing unexpected massive uterine bleeding in patients with cervical pregnancy, and it does not compromise future fertility [14]. However, this method has been shown to result in poor satisfaction when used to treat symptomatic adenomyosis [9].

Mean operating times have been reported as $20-56 \mathrm{~min}$ [9] with a hospital stay of 1-2 days [9, 12]. In Wang's series of women with menorrhagia and adenomyosis, the procedure resulted in $31 \%$ eumenorrhoea or hypomenorrhoea, 
and $81 \%$ achieved a reduction in menstrual loss by $30 \%$ or more.

Complications following this operation include postoperative pain. It has been reported that 24 out of 85 women who underwent uterine vessel ligation for the treatment of fibroids experienced lower abdominal pain postoperatively, which persisted for 2 weeks and mostly developed 5-6 days after surgery [10]. Another study also reported that procedure-related pain occurred in most women within the first $24 \mathrm{~h}$ of surgery, and up to $40.7 \%$ patients had nonmenstrual pain afterwards. This may be associated with target-tissue ischaemia [9]. Our patient did not experience debilitating postoperative pain.

Impaired ovarian function (transient amenorrhoea, menstrual irregularities) and premature ovarian failure after the procedure have been reported, especially in women older than 45 years. It is not known whether uterine vessel ligation alters the age at which ovarian activity ceases or what the long-term effect on the uterine blood supply is. This is a cause of concern, especially in women in whom there is the possibility of compromising subsequent fertility. However, successful pregnancies have been reported following uterine artery ligation, initially with laparotomy and more recently laparoscopy $[10,11]$. Our patient was counselled that there was no guarantee of a pregnancy following the surgery and that the long-term risks and benefits of the procedure are hard to predict.

A laparoscopic presacral neurectomy was not performed simultaneously at the time of the first procedure. It was thought that impairing the uterine perfusion could have had a beneficial effect on prostaglandin production. Likewise, some women experience uterine cramping as they pass clots, and it was postulated that if the procedure resulted in less bleeding it could have reduced the production of clots. Wang et al. [9] showed a positive effect on dysmenorrhoea, with $75 \%$ of their patients achieving "control" of it and $37.5 \%$ being free of analgaesia.

Laparoscopic bilateral uterine artery ligation in experienced hands is associated with low morbidity and has the potential to avoid the need for hysterectomy. The postoperative recovery is within days rather than weeks, which imparts considerable cost savings. We believe there is a place for laparoscopic uterine vessel ligation in the treatment of dysfunctional uterine bleeding. However, the long-term effects on symptomatic relief and the possible side effects of this technique must be fully assessed.

\section{References}

1. Bradley EA, Reidy JF, Forman RG, et al (1998) Transcatheter uterine artery embolisation to treat large uterine fibroids. $\mathrm{Br}$ J Obstet Gynaecol 105(2):235-240

2. Chen YJ, Wang PH, Yuan CC et al (2002) Successful pregnancy in a woman with symptomatic fibroids who underwent laparoscopic bipolar coagulation of uterine vessels. Fertil Steril 77(4):838-840

3. Waters EG (1952) Surgical management of postpartum hemorrhage with particular reference to ligation of uterine arteries. Am J Obstet Gynecol 64:1143

4. O'Leary JL, O'Leary JA (1966) Uterine artery ligation in the control of intractable postpartum hemorrhage. Am J Obstet Gynecol 94:920-924

5. O'Leary JL, O'Leary JA (1974) Uterine artery ligation for control of postcesarean section hemorrhage. Obstet Gynecol 43:849-853

6. Chou YC, Wang PH, Yuan CC et al (2002) Laparoscopic bipolar coagulation of uterine vessels to manage delayed postpartum hemorrhage. J Am Assoc Gynecol Laparosc 9 (4):541-544

7. Sapmaz E, Celik H, Altungul A (2003) Bilateral ascending uterine artery ligation vs. tourniquet use for hemostasis in cesarean myomectomy. A comparison. J Reprod Med 48 (12):950-954

8. Bateman W (1964) Treatment of intractable menorrhagia by bilateral uterine vessel interruption. Am J Obstet Gynecol 89 (6):825-827

9. Wang CJ, Yen CF, Lee CL et al (2002) Laparoscopic uterine artery ligation for treatment of symptomatic adenomyosis. J Am Assoc Gynecol Laparosc 9(3):293-296

10. Liu WM, Ng HT, Wu YC, et al (2001) Laparoscopic bipolar coagulation of uterine vessels: a new method for treating symptomatic fibroids. Fertil Steril 75:417-422

11. Mitchell GG, Mellor S, Burslem RW (1977) Pregnancy following bilateral uterine artery ligation. Br J Obstet Gynaecol $84: 551-554$

12. Lichtinger M, Hallson L, Calvo P et al (2002) Laparoscopic uterine artery occlusion for symptomatic leiomyomas. J Am Assoc Gynecol Laparosc 9(2):191-198

13. Park KH, Kim JY, Shin JS et al (2003) Treatment outcomes of uterine artery embolization and laparoscopic uterine artery ligation for uterine myoma. Yonsei Med J (Korea (South)) 44 (4):694-702

14. Lin H, Kung FT (2003) Combination of laparoscopic bilateral uterine artery ligation and intraamniotic methotrexate injection for conservative management of cervical pregnancy. J Am Assoc Gynecol Laparosc 10(2):215-218 